\title{
Postoperative C-reactive protein-to-albumin ratio predicts poor prognosis in patients with bladder cancer undergoing radial cystectomy
}

\author{
KENJI KURODA, SHINSUKE TASAKI, AKIO HORIGUCHI and KEIICHI ITO \\ Department of Urology, National Defense Medical College, Tokorozawa, Saitama 359-8513, Japan
}

Received September 12, 2020; Accepted November 18, 2020

DOI: $10.3892 / \mathrm{mco} .2021 .2216$

\begin{abstract}
The purpose of the present study was to investigate the prognostic value of the postoperative C-reactive protein/albumin ratio (CAR) in patients with bladder cancer undergoing radial cystectomy. The present study retrospectively reviewed 102 patients who underwent radical cystectomy and were followed for $\geq 6$ months postoperatively at our institution, and evaluated clinicopathological factors and laboratory parameters for cancer-specific survival (CSS) and extraurothelial recurrence-free survival (ERFS). Multivariate analysis using the Cox proportional hazards model revealed that only postoperative $\mathrm{CAR} \geq 0.27$ [hazard ratio (HR), 3.368; 95\% confidence interval $(\mathrm{CI}), 1.674-6.731 ; \mathrm{P}<0.001]$ was an independent factor for poor CSS rate. Higher postoperative CAR was also the only significant factor for shortened ERFS time (HR, 2.401; 95\% CI, 1.196-4.684; P=0.015). No significant association was identified between postoperative CAR $\geq 0.27$ and any pathological factors or postoperative laboratory markers besides postoperative neutrophil-to-lymphocyte ratio. Furthermore, postoperative CAR $(\geq 0.27)$ was an independent factor for poor CSS and ERFS rates in 48 patients with advanced $\mathrm{pT}$ stage $(\geq \mathrm{pT} 3)$ in the multivariate analysis $(\mathrm{P}=0.026$ and $\mathrm{P}=0.036$, respectively). A higher postoperative $\mathrm{CAR}$ value can provide additional information about the possibility of poor CSS and ERFS rates in patients with bladder cancer undergoing radical cystectomy.
\end{abstract}

\section{Introduction}

Bladder cancer is the tenth most common cancer in the world, with approximately 550,000 new cases and 200,000 deaths in 2018 (1). Approximately $25 \%$ of bladder cancer patients present with muscle-invasive bladder cancer at the time of

Correspondence to: Dr Kenji Kuroda, Department of Urology, National Defense Medical College, 3-2 Namiki, Tokorozawa, Saitama 359-8513, Japan

E-mail: kksmy@sa2.so-net.ne.jp

Key words: bladder cancer, radical cystectomy, postoperative marker, C-reactive protein, albumin diagnosis. This indicates a high possibility of metastasis and of affecting survival (2).

Radical cystectomy is an aggressive, conventional and standard therapeutic option for patients with muscle-invasive or bacillus Calmette-Guérin (BCG)-refractory non-muscle-invasive bladder cancer. Postoperative survival time has a high association with the pathological status at the time of radical cystectomy. In patients with stage II or III bladder carcinoma, the 5-year survival rates range from 50 to $80 \%$ (3-5). However, clinicopathological data alone has been insufficient for deciding the optimal treatment option (6). Based on poor surgical outcomes after radical cystectomy, the optimal postoperative monitoring option for high-risk bladder carcinoma is required.

There has been accumulating evidence supporting the rationale for the association of systemic inflammatory response (SIR) with tumor development and progression (7). Proinflammatory cytokines and growth factors are released into the systemic circulation as factors for SIR. In addition, it has been speculated that tumor growth factors expedite the release of cytokines from tissues. SIR may be activated secondary to local tissue damage caused by mutual responses between tumor necrosis factors and original cancer cells (8). These SIR markers include C-reactive protein (CRP), the Glasgow Prognostic Score (GPS), neutrophil-to-lymphocyte ratio (NLR), and platelet to lymphocyte ratio (PLR) (9-12). In addition, nutritional status, such as lower levels of serum albumin (Alb) and low body mass index, is associated with worse clinical outcomes postoperatively in patients with carcinoma, and CRP to Alb ratio (CAR), in combination with the systemic status of inflammation and nutrition, has been reported as an independent prognosticator in some types of carcinoma (13-16).

SIR markers have been measured preoperatively (13-16), but nutritional or inflammatory status needs to be stable before surgery or other invasive treatment. However, reports suggest postoperative SIR markers have potential prognostic significance, especially in patients with urothelial carcinoma. To the best of our knowledge, only a few studies have investigated the association of postoperative NLR and poor prognosis in patients with upper urinary tract and bladder urothelial carcinoma (17-19). Therefore, we sought to examine postoperative, as well as preoperative SIR markers, including CAR, to determine independent factors of poor prognosis in locally advanced bladder cancer. 
In the present study, we retrospectively investigated the clinicopathological and laboratory data of patients with bladder carcinoma who underwent radical cystectomy at National Defense Medical College (single center) to clarify whether postoperative CAR could be an independent prognostic factor for shorter time to cancer-specific death and/or extraurothelial recurrence.

\section{Patients and methods}

Patients. The medical records of 102 patients who underwent radical cystectomy between January 2007 and January 2020 and who were pathologically diagnosed with urothelial carcinoma before or at the time of cystectomy were retrospectively reviewed. They were followed for at least 6 months postoperatively at our institution. The study protocol (ID 2734) was accepted on June 14, 2017, by National Defense Medical College Ethics Committee, and an opt-out approach on the web page of the National Defense Medical College was used instead of collecting written informed consent from all participants.

A total of 80 males and 22 females with a median age of 71 years (range, $49-83$ years) were included in the present study. The median follow-up period after surgery was 38.9 months (range, 6.1-162.2 months). Table I shows additional clinicopathological and laboratory data. The absence of tumor from the histopathological examination of the cystectomy specimen is interpreted as pT0, which translated to 22 pT0 patients.

Extraurothelial recurrence after radical cystectomy indicates tumor recurrence outside the bladder or distant metastasis. In the present study, we defined recurrence-free survival as extraurothelial recurrence-free survival (ERFS).

The method of lymphadenectomy was the same as that described in earlier studies (20,21). All patients received regional lymphadenectomy, and 21 showed positive lymph node metastasis. Neoadjuvant chemotherapy was administered to 47 patients, 22 of whom developed postoperative recurrence or distant metastasis.

As part of postoperative chemotherapy, adjuvant chemotherapy was administered to 11 patients with histologically confirmed lymphovascular invasion (LVI) or pathologically determined pT3 or pT4 cancer, and salvage chemotherapy was administered to 26 patients who developed recurrence and/or distant metastasis after surgery. Both adjuvant and salvage chemotherapy were administered to 5 patients. The dose of cisplatin was adjusted according to the renal function of the patient.

All surgical specimens were processed according to standard pathological procedures and were histologically confirmed to be urothelial carcinoma with or without other tumor cell types. The pathological staging of the primary tumor (pT) was determined according to the American Joint Committee on Cancer TNM Classification (22), whereas tumor grading was determined according to the 2004 WHO classification of urothelial tumors (23). Tumor specimens were evaluated by two pathologists, and the patients were divided into two groups on the basis of the 2004 WHO classification system for tumor grading.

Each patient was monitored for local recurrence or distant metastasis every 3-6 months for the first 5 years after cystectomy and 6-12 months thereafter.
Estimated glomerular filtration rate and inflammatory indices. Preoperative laboratory tests were performed within 1 week before cystectomy, and postoperative laboratory tests was measured 1 to 2 months after surgery. In cases requiring postoperative chemotherapy, the postoperative blood examination was performed prior to postoperative chemotherapy. None of the patients enrolled in the present study had inflammatory diseases or hematological disorders at the time of blood tests.

The estimated glomerular filtration rate (eGFR) was calculated by computation using the following formula:

eGFR $\left(\mathrm{ml} / \mathrm{min} / 1.73 \mathrm{~m}^{2}\right)=194 x(\text { serum creatinine })^{-1.094} \mathrm{x}$ $\operatorname{Age}^{-0.287} \mathrm{x}(0.739$, for women $)$.

This formula is the isotope dilution mass spectrometry (IDMS)-traceable 4-variable Modification of Diet in Renal Disease (MDRD) Study equation, a modified equation from the IDMS MDRD Study with a coefficient derived from data by the Japanese Society of Nephrology-Chronic Kidney Disease Initiatives (JSN-CKDI). This formula has been reported to be more accurate for hospitalized Japanese patients with an eGFR of $<60 \mathrm{ml} / \mathrm{min} / 1.73 \mathrm{~m}^{2}$ than the original IDMS MDRD Study Equation (24).

Inflammatory indices were calculated as follows. CAR was calculated by dividing the CRP value (mg/dl) by the Alb value $(\mathrm{g} / \mathrm{dl})(10,14,15)$. The NLR was calculated by dividing the absolute neutrophil count by the absolute lymphocyte count $(9,19)$. The platelet to lymphocyte ratio (PLR) was calculated by dividing the absolute platelet count by the absolute lymphocyte count (11).

Statistical analysis. Univariate and multivariate analyses were performed using a Cox proportional hazards model to identify independent factors for shorter cancer-specific survival (CSS) and ERFS time. In addition, receiver operator characteristic (ROC) analysis was performed to determine the cut-off values of eGFR, CAR, NLR and PLR according to the method shown in earlier studies $(9,19,25)$. We also applied the cut-off values determined on the basis of CSS rate, according to previous reports $(9,19,25)$. Fisher's exact probability test was performed to examine the relationship between postoperative $\mathrm{CAR} \geq 0.27$ and any other pathological factors or postoperative laboratory markers. Survival curves were constructed using the Kaplan-Meier method, and the statistical differences among them were evaluated using the log-rank test. Statistical analyses were performed with JMP Pro 14 (SAS Institute). A P-value $<0.05$ was considered statistically significant.

\section{Results}

Independent factors for shortened CSS and ERFS time in all patients. ROC analysis revealed that patients with preoperative eGFR $\leq 46.88$, postoperative $\mathrm{eGFR} \leq 48.02$, preoperative $\mathrm{CAR} \geq 0.17$, postoperative CAR $\geq 0.27$, preoperative NLR $\geq 2.46$, postoperative $N L R \geq 2.19$, preoperative PLR $\geq 94.80$ and postoperative PLR $\geq 105.23$ had a higher association with cancer-specific death than patients without these conditions. As to eGFR, we considered $45 \mathrm{ml} / \mathrm{min} / 1.73 \mathrm{~m}^{2}$ was a practical threshold in the present study. The ROC curves for postoperative CAR for cancer-specific death and extraurothelial recurrence are shown in Fig. 1A and B. The postoperative CAR threshold was 0.27 for both CSS and ERFS. 
Table I. Clinicopathological and laboratory parameters.

\begin{tabular}{|c|c|}
\hline Parameters & Patien \\
\hline \multicolumn{2}{|l|}{ Clinicopathologic } \\
\hline \multicolumn{2}{|l|}{ Sex } \\
\hline \multicolumn{2}{|l|}{ Men } \\
\hline \multicolumn{2}{|l|}{ Women } \\
\hline \multicolumn{2}{|l|}{ Urine cytology } \\
\hline \multicolumn{2}{|l|}{ Positive } \\
\hline \multicolumn{2}{|l|}{ Negative } \\
\hline \multicolumn{2}{|l|}{ Unknown } \\
\hline \multicolumn{2}{|l|}{ Smoking history } \\
\hline \multicolumn{2}{|l|}{ Present } \\
\hline \multicolumn{2}{|l|}{ Absent } \\
\hline \multicolumn{2}{|l|}{ History of UTUC } \\
\hline \multicolumn{2}{|l|}{ Present } \\
\hline \multicolumn{2}{|l|}{ Absent } \\
\hline \multicolumn{2}{|c|}{ Recurrent or primary tumor } \\
\hline \multicolumn{2}{|l|}{ Recurrent } \\
\hline \multicolumn{2}{|l|}{ Primary } \\
\hline \multicolumn{2}{|l|}{ Reason for cystectomy } \\
\hline \multicolumn{2}{|l|}{ Muscle-invasive } \\
\hline \multicolumn{2}{|l|}{ BCG-refractory } \\
\hline \multicolumn{2}{|l|}{ Histology } \\
\hline \multicolumn{2}{|l|}{ UC alone } \\
\hline \multicolumn{2}{|l|}{ UC and other subtypes } \\
\hline \multicolumn{2}{|l|}{ No tumor } \\
\hline \multicolumn{2}{|l|}{ Pathological T stage } \\
\hline \multicolumn{2}{|l|}{$\geq \mathrm{T} 3$} \\
\hline$\leq \mathrm{T} 2$ & \\
\hline
\end{tabular}

Tumor grade

High

PUNLMP/low

Lymph node metastasis

Positive

Negative

Ureteral involvement

Positive

Negative

Surgical margins

Positive

Negative

Lymphovascular invasion

Positive

Negative

CIS

Positive

Negative

Laboratory

Preoperative eGFR, $\mathrm{ml} / \mathrm{min} / 1.73 \mathrm{~m}^{2}$
Table I. Continued.

\begin{tabular}{lc}
\hline Parameters & Patients, $\mathrm{n}$ \\
\hline Postoperative eGFR, $\mathrm{l} / \mathrm{min} / 1.73 \mathrm{~m}^{2}$ & \\
$\quad<45$ & 29 \\
$\quad \geq 45$ & 73 \\
Preoperative CAR & \\
$\geq 0.17$ & 32 \\
$<0.17$ & 70 \\
Postoperative CAR & \\
$\geq 0.27$ & 25 \\
$<0.27$ & 77 \\
Preoperative NLR & \\
$\geq 2.46$ & 45 \\
$<2.46$ & 57 \\
Postoperative NLR & \\
$\geq 2.19$ & 55 \\
$<2.19$ & 47 \\
Preoperative PLR & \\
$\geq 94.80$ & 83 \\
$<94.80$ & 19 \\
Postoperative PLR & \\
$\geq 105.23$ & \\
$<105.23$ & \\
\end{tabular}

UTUC, upper urinary tract urothelial carcinoma; BCG, bacille Calmette-Guérin; PUNLMP, papillary urothelial neoplasm of low malignant potential; CIS, carcinoma in situ; eGFR, estimated glomerular filtration rate; CAR, C-reactive protein $(\mathrm{mg} / \mathrm{dl})$ to albumin $(\mathrm{g} / \mathrm{dl})$ ratio; NLR, neutrophil to lymphocyte ratio; PLR, platelet to lymphocyte ratio.

A Cox proportional hazards model was performed to prove CAR was a more useful marker than CRP alone or Alb alone. On a univariate analysis, significantly shorter cancer-specific survival was associated with postoperative CRP $\geq 0.6$ [hazard ratio (HR), 3.249; 95\% confidence interval (CI), 1.713-6.204; $\mathrm{P}<0.001$ ], postoperative Alb $\leq 3.4$ [HR, 3.624; 95\% CI, 1.822-6.938; $\mathrm{P}<0.001]$, and postoperative $\mathrm{CAR} \geq 0.27$ [HR, 4.209; 95\% CI, 2.201-7.992; $\mathrm{P}<0.001]$. Therefore, postoperative CAR was used according to HR. Additionally, preoperative CAR was also used.

Another Cox proportional hazards model was constructed to detect the independent clinicopathological factors and laboratory parameters for shortened CSS time. Among these factors, in a univariate analysis, pT stage, tumor grade, LVI, preoperative eGFR, preoperative CAR, and postoperative CAR were found to be independent factors for shortened CSS time $(\mathrm{P}<0.001, \mathrm{P}=0.014, \mathrm{P}<0.001, \mathrm{P}=0.013, \mathrm{P}=0.017, \mathrm{P}<0.001$, respectively). Among these independent factors in univariate analysis, only postoperative CAR $(\geq 0.27)$ was an independent factor for worse CSS rate in the multivariate analysis [HR, 3.368; 95\% CI, 1.674-6.731; P<0.001] (Table II).

In another univariate analysis, ERFS time was found to be shorter in patients with higher pT stage, high tumor grade, lymph node metastasis, LVI, preoperative eGFR $<45$, 
A

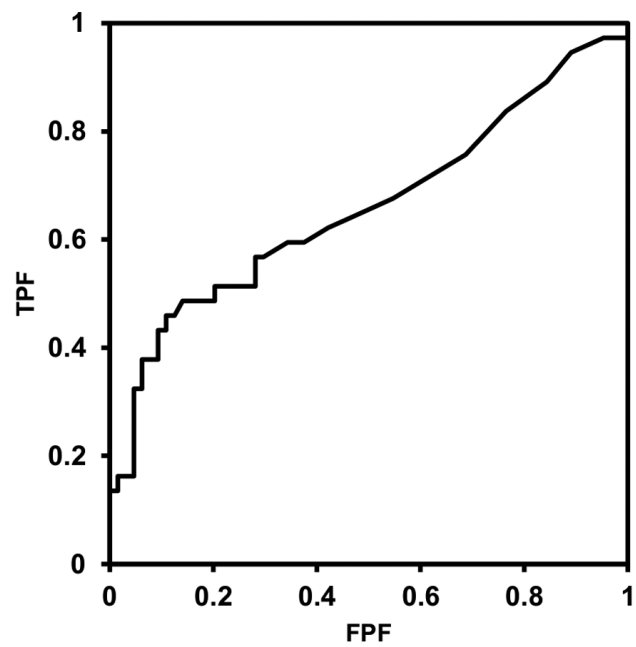

B

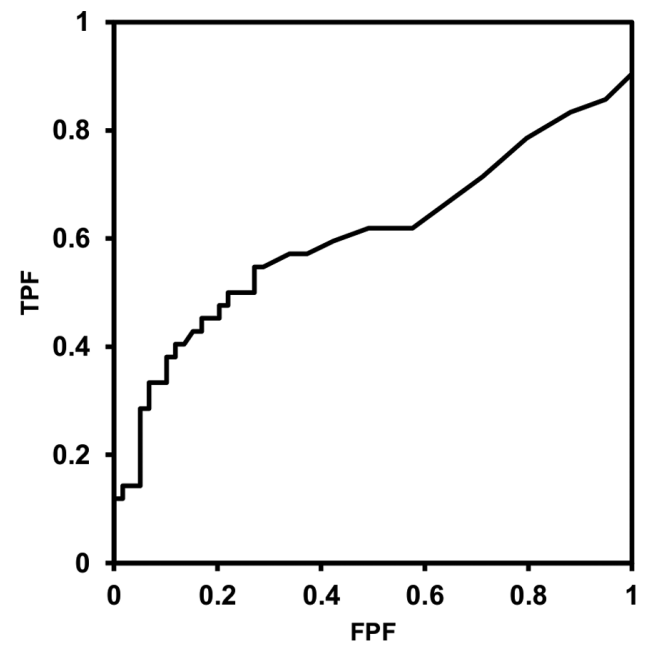

Figure 1. Receiver operating characteristic curves for postoperative C-reactive protein/albumin ratio for (A) cancer-specific mortality and (B) extraurothelial recurrence. The areas under the curves were (A) 0.667 and (B) 0.612 . TPF, true positive fraction; FPF, false positive fraction.

preoperative $\mathrm{CAR} \geq 0.17$, postoperative $\mathrm{CAR} \geq 0.27$, and preoperative $\mathrm{PLR} \geq 94.80(\mathrm{P}=0.002, \mathrm{P}=0.011, \mathrm{P}=0.039, \mathrm{P}=0.007$, $\mathrm{P}=0.015, \mathrm{P}=0.032, \mathrm{P}<0.001, \mathrm{P}=0.037$, respectively). Among these independent factors in univariate analysis, only postoperative $\mathrm{CAR} \geq 0.27$ was found to act as an independent factor for worse ERFS rate in the multivariate analysis (HR, 2.401; 95\% CI, 1.196-4.684; P=0.015) (Table III). Age, sex, body mass index, urine cytology, smoking history, history of upper urinary tract urothelial carcinoma, recurrent or primary tumor, reason for radical cystectomy, and the presence of histological subtypes were not independent predictors of worse CSS or ERFS rates in univariate analysis (data not shown).

Postoperative CAR $\geq 0.27$ was observed in 25 (24.5\%) patients. A statistically significant association was observed between postoperative CAR $\geq 0.27$ and postoperative NLR $\geq 2.19$ ( $\mathrm{P}=0.012$ ), but there was not any significant association between postoperative $\mathrm{CAR} \geq 0.27$ and any other pathological factors or postoperative laboratory markers (Table IV).

The Kaplan-Meier curves and the results of the log-rank test revealed a significant difference in the CSS and ERFS rates between all patients with and without postoperative CAR $\geq 0.27$ (both $\mathrm{P}<0.001$ ) (Fig. 2A and B).

Independent factors for shortened CSS and ERFS time in patients with advanced pT stage. Next, we sought to establish the independent factors of shorter CSS rates in patients with higher pT stage ( $\geq \mathrm{T} 3$ ). A Cox proportional hazards model was generated to determine the independent factors of shortened CSS rates among clinicopathological factors and laboratory parameters in 48 advanced pT stage $(\geq \mathrm{pT} 3)$ patients. In a univariate analysis, postoperative CAR and preoperative PLR were found to be independent factors for shortened CSS time $(\mathrm{P}=0.006, \mathrm{P}=0.038$, respectively). Among these independent factors in univariate analysis, only postoperative CAR $(\geq 0.27)$ was an independent factor for worse CSS rate in the multivariate analysis (HR, 2.600; 95\% CI, 1.126-5.833; $\mathrm{P}=0.026$ ) (Table V).

Another univariate analysis of Cox proportional hazards model revealed that ERFS time was found to be shorter in patients with postoperative $\mathrm{CAR} \geq 0.27$ and preoperative PLR $\geq 94.80$ ( $\mathrm{P}=0.012, \mathrm{P}=0.038$, respectively). Among these independent factors in univariate analysis, only postoperative CAR $\geq 0.27$ was found to act as an independent factor for worse ERFS rate in the multivariate analysis (HR, 2.399; 95\% CI, 1.062-5.297; P=0.036) (Table VI).

The Kaplan-Meier curves and the results of the log-rank test revealed significant differences in the CSS and ERFS rates between $\geq$ pT3 patients with and without postoperative CAR $\geq 0.27$ ( $\mathrm{P}=0.003, \mathrm{P}=0.006$, respectively) (Fig. $3 \mathrm{~A}$ and $\mathrm{B}$ ).

In total, $28(58.3 \%)$ patients with advanced pT stage $(\geq \mathrm{T} 3)$ received postoperative chemotherapy. Adjuvant chemotherapy was administered to 7 patients, and salvage chemotherapy was administered to 16 patients. Both adjuvant and salvage chemotherapy were administered to 5 patients. A total of 24 patients $(85.7 \%)$ received cisplatin plus gemcitabine, $2(7.1 \%)$ received docetaxel plus gemcitabine, and $2(7.1 \%)$ received carboplatin plus gemcitabine. The median number of chemotherapy cycles was three. Compared with those who underwent surgical treatment alone, patients with advanced $\mathrm{pT}$ stage ( $\geq$ T3) who received postoperative chemotherapy showed significantly worse CSS or ERFS rates $(\mathrm{P}=0.044, \mathrm{P}=0.005$, respectively) (Fig. 4A and B).

\section{Discussion}

In the present study, only postoperative $C A R \geq 0.27$ was an independent predictor of worse CSS as well as ERFS rates. Other laboratory parameters, such as eGFR, NLR, and PLR were found to be independent factors for shortened CSS time in univariate analyses but not independent factors in multivariate analyses. Although we added clinicopathological factors such as pathological $\mathrm{T}$ stage, tumor grade, lymph node metastasis, and LVI, postoperative CAR $\geq 0.27$ was designated as an only prognostic factor for worse CSS and ERFS rates in multivariate analysis of Cox proportional hazards model. Postoperative CAR $\geq 0.27$ did not have any significant association with pathological factors or postoperative laboratory parameters besides preoperative NLR, which means postoperative CAR is independent of pathological factors. We also performed multivariate analysis in patients with advanced pT stage $(\geq \mathrm{T} 3)$ and 
Table II. Univariate and multivariate analyses of independent factors for cancer-specific survival.

\begin{tabular}{|c|c|c|c|c|c|c|}
\hline \multirow[b]{2}{*}{ Parameters } & \multicolumn{3}{|c|}{ Univariate } & \multicolumn{3}{|c|}{ Multivariate } \\
\hline & Hazard ratio & $95 \% \mathrm{CI}$ & P-value & Hazard ratio & $95 \% \mathrm{CI}$ & P-value \\
\hline \multicolumn{7}{|l|}{ Clinicopathological } \\
\hline Pathological $\mathrm{T}$ stage $(\geq \mathrm{T} 3$ or $\leq \mathrm{T} 2)$ & 3.943 & $1.996-8.344$ & $<0.001$ & 2.149 & $0.974-5.070$ & 0.058 \\
\hline Tumor grade (high or PUNLMP/low) & 2.839 & $1.211-8.305$ & 0.014 & 2.363 & $0.930-7.277$ & 0.072 \\
\hline Lymph node metastasis (positive or negative) & 2.069 & $0.979-4.079$ & 0.056 & & & \\
\hline Ureter involvement (positive or negative) & 1.501 & $0.361-4.170$ & 0.524 & & & \\
\hline Surgical margin (positive or negative) & 1.405 & $0.419-3.530$ & 0.539 & & & \\
\hline Lymphovascular invasion (positive or negative) & 3.655 & $1.844-7.992$ & $<0.001$ & 1.615 & $0.712-3.942$ & 0.258 \\
\hline Carcinoma in situ (positive or negative) & 0.442 & $0.072-1.448$ & 0.203 & & & \\
\hline \multicolumn{7}{|l|}{ Laboratory } \\
\hline Preoperative eGFR $\left(<\right.$ or $\left.\geq 45 \mathrm{ml} / \mathrm{min} / 1.73 \mathrm{~m}^{2}\right)$ & 2.364 & $1.205-4.501$ & 0.013 & 1.972 & 0.949-3.982 & 0.068 \\
\hline Postoperative eGFR (< or $\left.\geq 45 \mathrm{ml} / \mathrm{min} / 1.73 \mathrm{~m}^{2}\right)$ & 1.767 & $0.874-3.407$ & 0.110 & & & \\
\hline Preoperative CAR ( $\geq$ or $<0.17$ ) & 2.240 & $1.163-4.254$ & 0.017 & 1.320 & $0.635-2.680$ & 0.451 \\
\hline Postoperative CAR $(\geq$ or $<0.27$ ) & 4.209 & $2.201-7.992$ & $<0.001$ & 3.368 & $1.674-6.731$ & $<0.001$ \\
\hline Preoperative NLR $(\geq$ or $<2.46$ ) & 1.863 & $0.977-3.606$ & 0.059 & & & \\
\hline Postoperative NLR ( $\geq$ or <2.19) & 1.667 & $0.872-3.309$ & 0.123 & & & \\
\hline Preoperative PLR $(\geq$ or $<94.80$ ) & 2.091 & 0.994-4.112 & 0.052 & & & \\
\hline Postoperative PLR ( $\geq$ or $<105.23$ ) & 1.419 & $0.606-2.950$ & 0.396 & & & \\
\hline
\end{tabular}

UTUC, upper urinary tract urothelial carcinoma; RC, radical cystectomy; BCG, bacillus Calmette-Guérin; PUNLMP, papillary urothelial neoplasm of low malignant potential; eGFR, estimated glomerular filtration rate ( $\left.\mathrm{ml} / \mathrm{min} / 1.73 \mathrm{~m}^{2}\right)$; CAR, C-reactive protein ( $\mathrm{mg} / \mathrm{dl}$ ) to albumin (g/dl) ratio; NLR, neutrophil to lymphocyte ratio; PLR, platelet to lymphocyte ratio.

Table III. Univariate and multivariate analyses of independent factors for extraurothelial recurrence-free survival.

\begin{tabular}{|c|c|c|c|c|c|c|}
\hline \multirow[b]{2}{*}{ Parameters } & \multicolumn{3}{|c|}{ Univariate } & \multicolumn{3}{|c|}{ Multivariate } \\
\hline & Hazard ratio & $95 \% \mathrm{CI}$ & P-value & Hazard ratio & $95 \% \mathrm{CI}$ & P-value \\
\hline \multicolumn{7}{|l|}{ Clinicopathological } \\
\hline Pathological $\mathrm{T}$ stage $(\geq \mathrm{T} 3$ or $\leq \mathrm{T} 2)$ & 2.602 & $1.412-4.954$ & 0.002 & 1.746 & $0.819-3.883$ & 0.151 \\
\hline Tumor grade (high or PUNLMP/low) & 2.724 & $1.238-7.187$ & 0.011 & 1.867 & $0.800-5.107$ & 0.156 \\
\hline Lymph node metastasis (positive or negative) & 2.114 & $1.041-4.021$ & 0.039 & 1.664 & $0.764-3.466$ & 0.194 \\
\hline Ureter involvement (positive or negative) & 1.858 & $0.557-4.622$ & 0.278 & & & \\
\hline Surgical margin (positive or negative) & 1.239 & $0.372-3.079$ & 0.692 & & & \\
\hline Lymphovascular invasion (positive or negative) & 2.323 & $1.256-4.469$ & 0.007 & 1.050 & $0.486-2.346$ & 0.902 \\
\hline Carcinoma in situ (positive or negative) & 0.844 & $0.253-2.099$ & 0.741 & & & \\
\hline \multicolumn{7}{|l|}{ Laboratory parameters } \\
\hline Preoperative eGFR $\left(<\right.$ or $\geq 45 \mathrm{ml} / \mathrm{min} / 1.73 \mathrm{~m}^{2}$ ) & 2.209 & $1.173-4.055$ & 0.015 & 1.699 & $0.858-3.276$ & 0.126 \\
\hline Postoperative eGFR $\left(<\right.$ or $\left.\geq 45 \mathrm{ml} / \mathrm{min} / 1.73 \mathrm{~m}^{2}\right)$ & 1.586 & $0.824-2.933$ & 0.162 & & & \\
\hline Preoperative CAR $(\geq$ or $<0.17$ ) & 1.987 & $1.065-3.637$ & 0.032 & 1.327 & $0.651-2.623$ & 0.427 \\
\hline Postoperative CAR ( $\geq$ or $<0.27$ ) & 3.147 & $1.684-5.766$ & $<0.001$ & 2.401 & $1.196-4.684$ & 0.015 \\
\hline Preoperative NLR ( $\geq$ or $<2.46$ ) & 1.504 & $0.823-2.756$ & 0.183 & & & \\
\hline Postoperative NLR ( $\geq$ or $<2.19$ ) & 1.597 & $0.870-3.030$ & 0.132 & & & \\
\hline Preoperative PLR $(\geq$ or $<94.80$ ) & 2.133 & $1.049-4.060$ & 0.037 & 1.888 & $0.868-3.886$ & 0.106 \\
\hline Postoperative PLR ( $\geq$ or $<105.23$ ) & 1.407 & $0.633-2.814$ & 0.379 & & & \\
\hline
\end{tabular}

UTUC, upper urinary tract urothelial carcinoma; RC, radical cystectomy; BCG, bacillus Calmette-Guérin; PUNLMP, papillary urothelial neoplasm of low malignant potential; eGFR, estimated glomerular filtration rate ( $\left.\mathrm{ml} / \mathrm{min} / 1.73 \mathrm{~m}^{2}\right)$; CAR, C-reactive protein ( $\mathrm{mg} / \mathrm{dl}$ ) to albumin (g/dl) ratio; NLR, neutrophil to lymphocyte ratio; PLR, platelet to lymphocyte ratio. 
Table IV. Association between postoperative CAR and clinicopathological parameters.

\begin{tabular}{|c|c|c|c|c|}
\hline \multirow[b]{2}{*}{ Parameters } & \multirow[b]{2}{*}{ Total $(\%)(n=102)$} & \multicolumn{2}{|c|}{ Postoperative CAR } & \multirow[b]{2}{*}{ P-value } \\
\hline & & $\geq 0.27, \mathrm{n}(\%)$ & $<0.27, \mathrm{n}(\%)$ & \\
\hline \multicolumn{5}{|c|}{ Pathological T stage } \\
\hline$\geq \mathrm{T} 3$ & $48(47.1)$ & $14(29.2)$ & $34(70.8)$ & \multirow[t]{2}{*}{0.360} \\
\hline$\leq \mathrm{T} 2$ & $54(52.9)$ & $11(20.4)$ & $43(79.6)$ & \\
\hline \multicolumn{5}{|l|}{ Tumor grade } \\
\hline High & $75(73.5)$ & $19(25.3)$ & $56(74.7)$ & \multirow[t]{2}{*}{$>0.999$} \\
\hline PUNLMP/Low & $27(26.5)$ & $6(22.2)$ & $21(77.8)$ & \\
\hline \multicolumn{5}{|c|}{ Lymph node metastasis } \\
\hline Positive & $21(20.6)$ & $7(33.3)$ & $14(66.7)$ & \multirow[t]{2}{*}{0.393} \\
\hline Negative & $81(79.4)$ & $18(22.2)$ & $63(77.8)$ & \\
\hline \multicolumn{5}{|c|}{ Lymphovascular invasion } \\
\hline Positive & $50(49.0)$ & $15(30.0)$ & $35(70.0)$ & \multirow[t]{2}{*}{0.253} \\
\hline Negative & $52(51.0)$ & $10(19.2)$ & $42(80.8)$ & \\
\hline \multicolumn{5}{|c|}{ Postoperative eGFR, $\mathrm{ml} / \mathrm{min} / 1.73 \mathrm{~m}^{2}$} \\
\hline$<45$ & $29(28.4)$ & $9(31.0)$ & $20(69.0)$ & \multirow[t]{2}{*}{0.444} \\
\hline$\geq 45$ & $73(71.6)$ & $16(21.9)$ & $57(78.1)$ & \\
\hline \multicolumn{5}{|l|}{ Postoperative NLR } \\
\hline$\geq 2.19$ & $55(53.9)$ & $19(34.5)$ & $36(65.5)$ & \multirow[t]{2}{*}{0.012} \\
\hline$<2.19$ & $47(46.1)$ & $6(12.8)$ & $41(87.2)$ & \\
\hline \multicolumn{5}{|l|}{ Postoperative PLR } \\
\hline$\geq 105.23$ & $85(83.3)$ & $20(23.5)$ & $65(76.5)$ & \multirow[t]{2}{*}{0.758} \\
\hline$<105.23$ & $17(16.7)$ & $5(29.4)$ & $12(70.6)$ & \\
\hline
\end{tabular}

CAR, C-reactive protein $(\mathrm{mg} / \mathrm{dl})$ to albumin $(\mathrm{g} / \mathrm{dl})$ ratio; PUNLMP, papillary urothelial neoplasm of low malignant potential; eGFR, estimated glomerular filtration rate $\left(\mathrm{ml} / \mathrm{min} / 1.73 \mathrm{~m}^{2}\right)$; NLR, neutrophil to lymphocyte ratio; PLR, platelet to lymphocyte ratio.
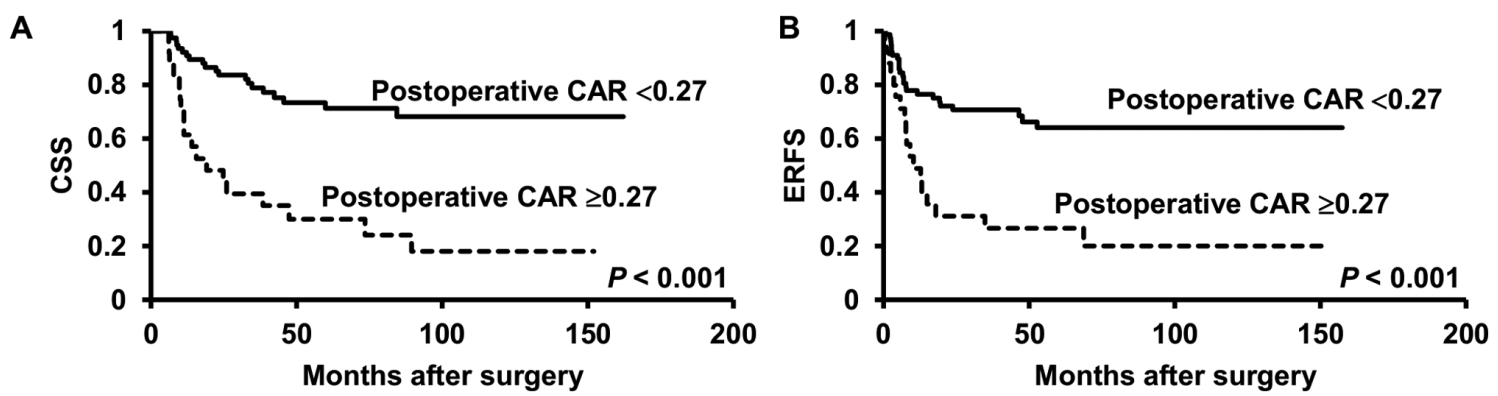

Figure 2. Survival analysis based on postoperative CAR values. There was a significant difference in time to (A) cancer-specific mortality and (B) extraurothelial recurrence between patients with and without postoperative CAR $\geq 0.27$. CSS, cancer-specific survival; ERFS, extraurothelial recurrence-free survival; CAR, C-reactive protein/albumin ratio.

found that higher postoperative CAR was the only independent predictor of poor CSS (HR, 2.600; $95 \%$ CI, 1.126-5.833; $\mathrm{P}=0.026)$ and ERFS rates (HR, 2.399; 95\% CI, 1.062-5.297; $\mathrm{P}=0.036$ ). We also found that postoperative chemotherapy did not improve prognosis in patients with advanced pT stage. This may be because 21 out of 28 patients $(75 \%)$ received salvage chemotherapy, and a higher postoperative CAR could facilitate monitoring of patients with $\geq \mathrm{pT} 3$ after radical cystectomy.

CIS at radical cystectomy is known to increase the risk of recurrence, especially in organ-confined patients (26-28). However, out of 10 patients with CIS, two had advanced pT stage
( $\geq \mathrm{T} 3$ ), one showed LVI, and other two had higher postoperative CAR $(\geq 0.27)$ in the present study. This may explain why patients with CIS did not show worse survival in this study.

Numerous studies have demonstrated an association between preoperative NLR and survival $(9,29,30)$. Conversely, some studies investigated whether postoperative SIR markers could be an effective prognostic marker for patients with clinically localized upper urinary tract urothelial carcinoma undergoing radical nephroureterectomy $(19,31)$. Subsequently, postoperative NLR has been suggested as a beneficial prognostic marker not only in urothelial carcinoma $(17,18)$, but also 
Table V. Univariate and multivariate analyses of independent factors for cancer-specific survival in 48 patients with advanced pT stage $(\geq \mathrm{T} 3)$.

\begin{tabular}{|c|c|c|c|c|c|c|}
\hline \multirow[b]{2}{*}{ Parameters } & \multicolumn{3}{|c|}{ Univariate } & \multicolumn{3}{|c|}{ Multivariate } \\
\hline & Hazard ratio & $95 \% \mathrm{CI}$ & $\mathrm{P}$-value & Hazard ratio & $95 \% \mathrm{CI}$ & P-value \\
\hline \multicolumn{7}{|l|}{ Clinicopathological } \\
\hline Tumor grade (high or PUNLMP/low) & 1.527 & $0.447-9.570$ & 0.546 & & & \\
\hline Lymph node metastasis (positive or negative) & 1.204 & $0.529-2.599$ & 0.647 & & & \\
\hline Ureter involvement (positive or negative) & 1.164 & $0.346-7.239$ & 0.833 & & & \\
\hline Surgical margin (positive or negative) & 1.069 & $0.410-3.653$ & 0.902 & & & \\
\hline Lymphovascular invasion (positive or negative) & 1.822 & $0.636-7.675$ & 0.291 & & & \\
\hline Carcinoma in situ (positive or negative) & 0.729 & $0.041-3.467$ & 0.745 & & & \\
\hline \multicolumn{7}{|l|}{ Laboratory } \\
\hline Preoperative eGFR $\left(<\right.$ or $\left.\geq 45 \mathrm{ml} / \mathrm{min} / 1.73 \mathrm{~m}^{2}\right)$ & 1.848 & $0.846-3.951$ & 0.121 & & & \\
\hline Postoperative eGFR $\left(<\right.$ or $\left.\geq 45 \mathrm{ml} / \mathrm{min} / 1.73 \mathrm{~m}^{2}\right)$ & 1.434 & $0.631-3.087$ & 0.376 & & & \\
\hline Preoperative CAR $(\geq$ or $<0.17$ ) & 1.843 & $0.854-3.952$ & 0.118 & & & \\
\hline Postoperative CAR $(\geq$ or $<0.27$ ) & 3.067 & $1.398-6.588$ & 0.006 & 2.600 & $1.126-5.833$ & 0.026 \\
\hline Preoperative NLR ( $\geq$ or $<2.46$ ) & 1.270 & $0.592-2.826$ & 0.541 & & & \\
\hline Postoperative NLR ( $\geq$ or $<2.19$ ) & 1.521 & $0.706-3.456$ & 0.287 & & & \\
\hline Preoperative PLR ( $\geq$ or $<94.80$ ) & 2.586 & $1.059-5.761$ & 0.038 & 1.841 & $0.231-1.381$ & 0.190 \\
\hline Postoperative PLR ( $\geq$ or $<105.23$ ) & 1.489 & $0.545-3.485$ & 0.409 & & & \\
\hline
\end{tabular}

UTUC, upper urinary tract urothelial carcinoma; RC, radical cystectomy; BCG, bacillus Calmette-Guérin; PUNLMP, papillary urothelial neoplasm of low malignant potential; eGFR, estimated glomerular filtration rate ( $\left.\mathrm{ml} / \mathrm{min} / 1.73 \mathrm{~m}^{2}\right)$; CAR, C-reactive protein ( $\mathrm{mg} / \mathrm{dl}$ ) to albumin (g/dl) ratio; NLR, neutrophil to lymphocyte ratio; PLR, platelet to lymphocyte ratio.

Table VI. Univariate and multivariate analyses of independent factors for extraurothelial recurrence-free survival in 48 patients with advanced pT stage $(\geq \mathrm{T} 3)$.

\begin{tabular}{|c|c|c|c|c|c|c|}
\hline \multirow[b]{2}{*}{ Parameters } & \multicolumn{3}{|c|}{ Univariate } & \multicolumn{3}{|c|}{ Multivariate } \\
\hline & Hazard ratio & $95 \% \mathrm{CI}$ & P-value & Hazard ratio & $95 \% \mathrm{CI}$ & P-value \\
\hline \multicolumn{7}{|l|}{ Clinicopathological } \\
\hline Tumor grade (high or PUNLMP/low) & 2.560 & $0.542-45.733$ & 0.284 & & & \\
\hline Lymph node metastasis (positive or negative) & 1.560 & $0.702-3.342$ & 0.266 & & & \\
\hline Ureter involvement (positive or negative) & 1.051 & $0.313-6.536$ & 0.945 & & & \\
\hline Surgical margin (positive or negative) & 1.050 & $0.403-3.582$ & 0.928 & & & \\
\hline Lymphovascular invasion (positive or negative) & 1.770 & $0.616-7.465$ & 0.317 & & & \\
\hline Carcinoma in situ (positive or negative) & 1.121 & $0.063-5.302$ & 0.913 & & & \\
\hline \multicolumn{7}{|l|}{ Laboratory } \\
\hline Preoperative eGFR $\left(<\right.$ or $\left.\geq 45 \mathrm{ml} / \mathrm{min} / 1.73 \mathrm{~m}^{2}\right)$ & 1.736 & $0.793-3.727$ & 0.164 & & & \\
\hline Postoperative eGFR $\left(<\right.$ or $\geq 45 \mathrm{ml} / \mathrm{min} / 1.73 \mathrm{~m}^{2}$ ) & 1.315 & $0.579-2.830$ & 0.499 & & & \\
\hline Preoperative CAR $(\geq$ or $<0.17$ ) & 1.329 & $0.599-2.841$ & 0.473 & & & \\
\hline Postoperative CAR $(\geq$ or $<0.27$ ) & 2.777 & $1.265-5.971$ & 0.012 & 2.399 & $1.062-5.297$ & 0.036 \\
\hline Preoperative NLR ( $\geq$ or $<2.46$ ) & 1.004 & $0.469-2.165$ & 0.992 & & & \\
\hline Postoperative NLR $(\geq$ or $<2.19$ ) & 1.711 & $0.786-4.003$ & 0.179 & & & \\
\hline Preoperative PLR $(\geq$ or $<94.80$ ) & 2.613 & $1.061-5.902$ & 0.038 & 2.058 & $0.208-1.230$ & 0.122 \\
\hline Postoperative PLR $(\geq$ or $<105.23$ ) & 1.416 & $0.518-3.316$ & 0.469 & & & \\
\hline
\end{tabular}

UTUC, upper urinary tract urothelial carcinoma; RC, radical cystectomy; BCG, bacillus Calmette-Guérin; PUNLMP, papillary urothelial neoplasm of low malignant potential; eGFR, estimated glomerular filtration rate ( $\left.\mathrm{ml} / \mathrm{min} / 1.73 \mathrm{~m}^{2}\right)$; CAR, C-reactive protein ( $\mathrm{mg} / \mathrm{dl}$ ) to albumin (g/dl) ratio; NLR, neutrophil to lymphocyte ratio; PLR, platelet to lymphocyte ratio. 
A

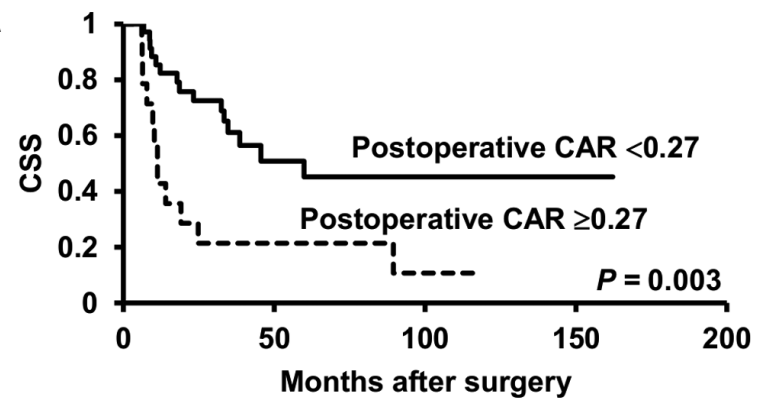

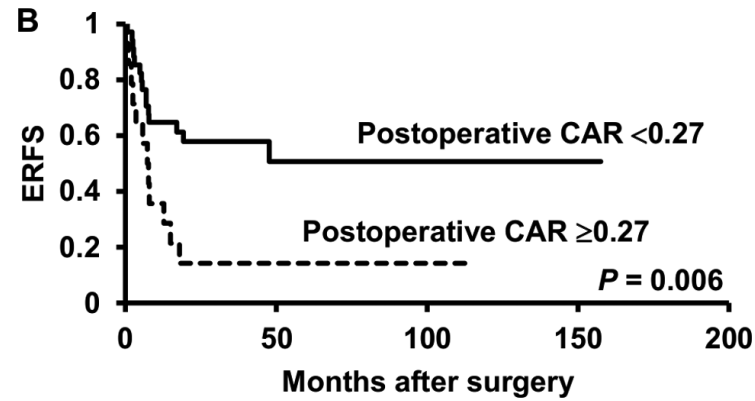

Figure 3. Survival analysis based on postoperative CAR of 48 patients with advanced $\mathrm{pT}$ stage $(\geq \mathrm{T} 3)$. There was a significant difference in time to (A) cancer-specific mortality and (B) extraurothelial recurrence between patients with and without postoperative CAR $\geq 0.27$. CSS, cancer-specific survival; ERFS, extraurothelial recurrence-free survival; CAR, C-reactive protein/albumin ratio.

A

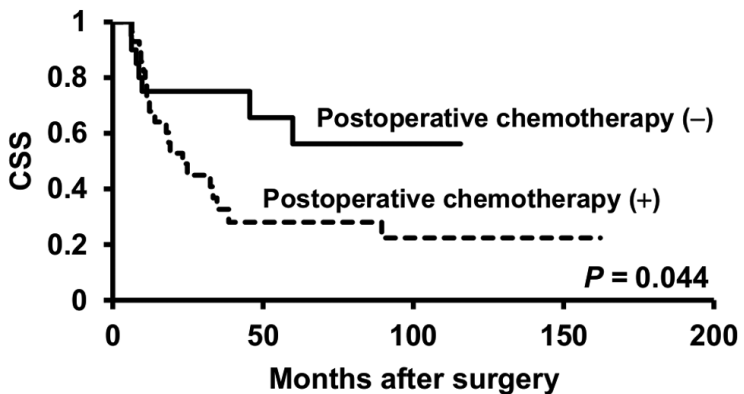

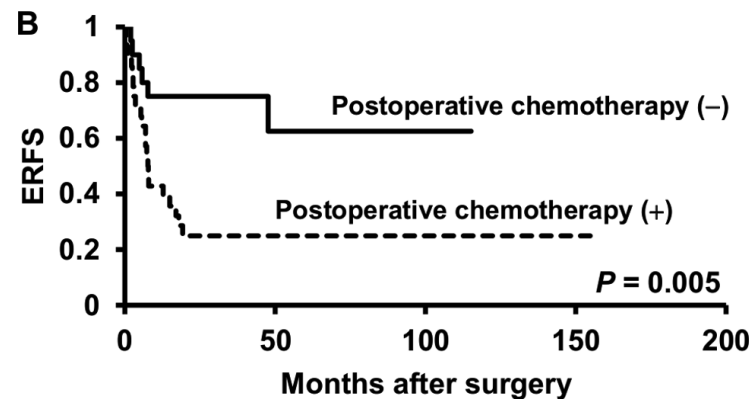

Figure 4. Survival curves of 48 patients with advanced pT stage ( $\geq \mathrm{T} 3)$ who received postoperative chemotherapy. There was a significant difference in time to (A) cancer-specific mortality and (B) extraurothelial recurrence between patients with and without postoperative chemotherapy. CSS, cancer-specific survival; ERFS, extraurothelial recurrence-free survival.

in other carcinomas $(32,33)$. Preoperative NLR only reflects the balance host protumor inflammatory status and antitumor immune status before surgery, whereas postoperative NLR indicates the balance between tumor inflammatory response and host immune response after surgical removal of the tumor, which should provide a more precise indication of treatment response (33).

On a similar note to papers reporting on the prognostic value of NLR, several studies have investigated presurgical or pretreatment CAR as a predictor of poor prognosis in patients with several types of tumors (14-16). The prognostic value of postoperative CAR has remained unclear in patients with bladder cancer undergoing radical cystectomy, although several reports have implied clinical significance of postoperative CRP $(31,34)$. The present study demonstrated that high postoperative CAR levels were significantly associated with poor prognosis in patients with locally advanced bladder cancer. Patients with higher postoperative CAR also exhibited a significantly worse prognosis compared with those with lower postoperative CAR, even among those with advanced pT stage ( $\geq \mathrm{T} 3)$. Based on this finding and in relation to earlier suggestions $(19,33)$, this may be because higher postoperative CAR possibly indicates potential residual cancer, including micrometastases, whereas preoperative CAR values indicate the presence of the primary tumor and potential micrometastatic lesions. This is the reason why higher preoperative CAR is an independent factor only in univariate analysis, whereas higher postoperative CAR is an independent predictor of worse CSS and ERFS rates in multivariate analysis. Thus, if postoperative CAR reflects micrometastases, it is potentially a significant prognostic marker in patients with locally advanced bladder cancer undergoing surgical treatment.

Approximately $20 \%$ of all cancer-related deaths are attributable to malnutrition (35). In addition, malnutrition is present in $40-80 \%$ of all patients with cancer at some stage during the clinical course of their disease (35). Malnutrition and inflammation suppress the synthesis of serum Alb, which is an indicator of the nutritional status of patients, as well as the severity, progression, and prognosis of the disease (36). Serum Alb is an independent predictor of clinical outcomes in various cancers $(37,38)$. Moreover, many studies have combined CRP and Alb to create new SIR markers, such as GPS and CAR. In fact, both GPS and CAR are independent indicators of poor survival in various types of cancer (12,14-16). In the present study, CAR was applied as a variable because CAR is a continuous value compared with GPS, which is a dichotomous variable, although it was required to determine the cut-off values for cancer-specific death and/or extraurothelial recurrence.

This study has some potential limitations. First, our sample size was relatively small, although the CSS and ERFS rates were evaluated as our clinical endpoint. With a longer follow-up and a larger sample population, the statistical strength of our study can be reinforced, leading to more precise prognosis. Second, we could not clarify the background between postoperative CAR and worse survival rates in patients with advanced bladder cancer, although the possible implications for patients with a higher postoperative CAR value are suggested. Third, several other factors that may have influenced the inflammatory status, such as diabetes mellitus and/or hyperlipidemia, were not included in the present study. However, we deemed 
these conditions had only minimal influence on the values of inflammatory indices because body mass index did not have any significant association with prognosis (data not shown). Notwithstanding these limitations, our data indicate that a higher postoperative CAR can be an independent prognostic factor for worse survival rates.

In summary, a higher postoperative CAR value can provide additional information about the possibility of worse CSS and ERFS rates.

In conclusion, among the patients with bladder cancer undergoing radical cystectomy, survival rates are worse in those with higher postoperative CAR values than in those with lower postoperative CAR values. Therefore, patients with higher postoperative CAR should undergo additional treatment or at least careful follow-up.

\section{Acknowledgements}

Not applicable.

\section{Funding}

No funding was received.

\section{Availability of data and materials}

The datasets used and/or analyzed during the current study are available from the corresponding author on reasonable request.

\section{Authors' contributions}

$\mathrm{KK}, \mathrm{ST}, \mathrm{AH}$ and $\mathrm{KI}$ were involved in the conception and design of the study. KK, ST, AH and KI collected the data, and $\mathrm{KK}$ analyzed the data. KK drafted the manuscript. KK and KI reviewed and edited the manuscript. All authors read and approved the final manuscript.

\section{Ethics approval and consent to participate}

All procedures performed in the studies were in accordance with the ethical standards of the National Defense Medical College (Saitama, Japan; ID 2734). The study protocol was accepted on June 14, 2017, by the National Defense Medical College Ethics Committee, and an opt-out approach on the web page of the National Defense Medical College was used instead of collecting written informed consent from all participants.

\section{Patient consent for publication}

Not applicable.

\section{Competing interests}

The authors declare that they have no competing interests.

\section{References}

1. Bray F, Ferlay J, Soerjomataram I, Siegel RL, Torre LA and Jemal A: Global cancer statistics 2018: GLOBOCAN estimates of incidence and mortality worldwide for 36 cancers in 185 countries. CA Cancer J Clin 68: 394-424, 2018.
2. National collaborating centre for cancer (UK): Bladder cancer: Diagnosis and management, 2015.

3. Shariat SF, Karakiewicz PI, Palapattu GS, Lotan Y, Rogers CG Amiel GE, Vazina A, Gupta A, Bastian PJ, Sagalowsky AI, et al: Outcomes of radical cystectomy for transitional cell carcinoma of the bladder: A contemporary series from the Bladder Cancer Research Consortium. J Urol 176: 2414-2422, 2006.

4. Hautmann RE, de Petriconi RC, Pfeiffer $C$ and Volkmer BG: Radical cystectomy for urothelial carcinoma of the bladder without neoadjuvant or adjuvant therapy: Long-term results in 1100 patients. Eur Urol 61: 1039-1047, 2012.

5. Stein JP, Lieskovsky G, Cote R, Groshen S, Feng AC, Boyd S, Skinner E, Bochner B, Thangathurai D, Mikhail M, et al: Radical cystectomy in the treatment of invasive bladder cancer: Long-term results in 1,054 patients. J Clin Oncol 19: 666-675, 2001.

6. Canter D, Long C, Kutikov A, Plimack E, Saad I, Oblaczynski M, Zhu F, Viterbo R, Chen DY, Uzzo RG, et al: Clinicopathological outcomes after radical cystectomy for clinical T2 urothelial carcinoma: Further evidence to support the use of neoadjuvant chemotherapy. BJU Int 107: 58-62, 2011.

7. Mantovani A, Allavena P, Sica A and Balkwill F: Cancer-related inflammation. Nature 454: 436-444, 2008.

8. Scott HR, McMillan DC, Forrest LM, Brown DJ, McArdle CS and Milroy R: The systemic inflammatory response, weight loss, performance status and survival in patients with inoperable non-small cell lung cancer. Br J Cancer 87: 264-267, 2002.

9. Kang M, Jeong CW, Kwak C, Kim HH and Ku JH: Preoperative neutrophil-lymphocyte ratio can significantly predict mortality outcomes in patients with non-muscle invasive bladder cancer undergoing transurethral resection of bladder tumor. Oncotarget 8: 12891-12901, 2016.

10. Omae K, Kondo T and Tanabe K: High preoperative C-reactive protein values predict poor survival in patients on chronic hemodialysis undergoing nephrectomy for renal cancer. Urol Oncol 33: e9-e13, 2015.

11. Gunduz S, Mutlu H, Tural D, Yildiz O, Uysal M, Coskun HS and Bozcuk H: Platelet to lymphocyte ratio as a new prognostic for patients with metastatic renal cell cancer. Asia Pac J Clin Oncol 11: 288-292, 2015.

12. Yuksel OH, Akan S, Uukmez A, Yildirim C, Sahin A and Verit A: Preoperative Glasgow prognostic score as a predictor of primary bladder cancer recurrence. Mol Clin Oncol 5: 201-206, 2016.

13. Morgan TM, Tang D, Stratton KL, Barocas DA, Anderson CB, Gregg JR, Chang SS, Cookson MS, Herrell SD, Smith JA Jr and Clark PE: Preoperative nutritional status is an important predictor of survival in patients undergoing surgery for renal cell carcinoma. Eur Urol 59: 923-928, 2011.

14. Zhou T, Zhan J, Hong S, Hu Z, Fang W, Qin T, Ma Y, Yang Y, He X, Zhao Y, et al: Ratio of C-reactive protein/albumin is an inflammatory prognostic score for predicting overall survival of patients with small-cell lung cancer. Sci Rep 5: 10481, 2015.

15. Liu X, Sun X, Liu J, Kong P, Chen S, Zhan Y and Xu D: Preoperative $\mathrm{C}$-reactive protein/albumin ratio predicts prognosis of patients after curative resection for gastric cancer. Transl Oncol 8: 339-345, 2015.

16. Kinoshita A, Onoda H, Imai N, Iwaku A, Oishi M, Tanaka K, Fushiya N, Koike K, Nishino $H$ and Matsushima M: The C-reactive protein/albumin ratio, a novel inflammation-based prognostic score, predicts outcomes in patients with hepatocellular carcinoma. Ann Surg Oncol 22: 803-810, 2015.

17. Morizawa Y, Miyake M, Shimada K, Hori S, Tatsumi Y, Nakai Y, Anai S, Tanaka N, Konishi $\mathrm{N}$ and Fujimoto K: Neutrophil-to-lymphocyte ratio as a detection marker of tumor recurrence in patients with muscle-invasive bladder cancer after radical cystectomy. Urol Oncol Semin Orig Investig 34: 257. e11-e17, 2016.

18. Kang M, Jeong CW, Kwak C, Kim HH and Ku JH: The prognostic significance of the early postoperative neutrophil-to-lymphocyte ratio in patients with urothelial carcinoma of the bladder undergoing radical cystectomy. Ann Surg Oncol 23: 335-342, 2016.

19. Nishihara K, Suekane S, Ueda K, Nakiri M, Matsuo M and Igawa T: High postoperative neutrophil-to-lymphocyte ratio as a poorprognostic marker in patients with upper tract urothelial carcinoma. Oncol Lett 17: 5241-5250, 2019.

20. Herr HW: Extent of pelvic lymph node dissection during radical cystectomy: Where and Why! Eur Urol 57: 212-213, 2010.

21. Ghoneim MA, Abdel-Latif M, El-Mekresh M, Abol-Enein H, Mosbah A, Ashamallah A and El-Baz MA: Radical cystectomy for carcinoma of the bladder: 2,720 consecutive cases 5 years later. J Urol 180: 121-127, 2008. 
22. Sobin L, Gospodarowicz M and Wittekind C: TNM classification of malignant tumours, 7th edition, 2009.

23. John E, Guido S, Jonathan E and Isabell S: Pathology and genetics: Tumours of the urinary system and male genital system, 2004.

24. Imai E, Horio M, Nitta K, Yamagata K, Iseki K, Tsukamoto Y, Ito S, Makino H, Hishida A and Matsuo S: Modification of the modification of diet in renal disease (MDRD) Study equation for Japan. Am J Kidney Dis 50: 927-937, 2007.

25. Shen Y, Wang H, Li W and Chen J: Prognostic significance of the CRP/Alb and neutrophil to lymphocyte ratios in hepatocellular carcinoma patients undergoing TACE and RFA. J Clin Lab Anal 33: e22999, 2019.

26. Utz DC, Hanash KA and Farrow GM: The plight of the patient with carcinoma in situ of the bladder. J Urol 103: 160-164, 1970.

27. Stanisic TH, Donovan JM, Lebouton J and Graham AR: 5-Year experience with intravesical therapy of carcinoma in situ: An inquiry into the risks of 'conservative' management. J Urol 138: $1158-1161,1987$.

28. Lamm D, Herr H, Jakse G, Kuroda M, Mostofi FK, Okajima E, Sakamoto A, Sesterhenn I and da Silva FC: Updated concepts and treatment of carcinoma in situ. Urol Oncol 4: 130-138, 1998

29. Uemura K, Kawahara T, YamashitaD, Jikuya R, Abe K, Tatenuma T, Yokomizo Y, Izumi K, Teranishi JI, Makiyama K, et al: Neutrophil-to-lymphocyte ratio predicts prognosis in castration-resistant prostate cancer patients who received cabazitaxel chemotherapy. Biomed Res Int 2017: 7538647, 2017.

30. Kasuga J, Kawahara T, Takamoto D, Fukui S, Tokita T, Tadenuma T, Narahara M, Fusayasu S, Terao H, Izumi K, et al: Increased neutrophil-to-lymphocyte ratio is associated with disease-specific mortality in patients with penile cancer. BMC Cancer 16: 396, 2016

31. Tanaka N, Kikuchi E, Shirotake S, Kanao K, Matsumoto K, Kobayashi H, Miyazaki Y, Ide H, Obata J, Hoshino K, et al: The predictive value of $\mathrm{C}$-reactive protein for prognosis in patients with upper tract urothelial carcinoma treated with radical nephroureterectomy: A multi-institutional study. Eur Urol 65: 227-234, 2014
32. Tanaka H, Tamura T, Toyokawa T, Muguruma K, Miki Y, Kubo N, Sakurai K, Hirakawa K and Ohira M: Clinical relevance of postoperative neutrophil-lymphocyte ratio (NLR) to recurrence after adjuvant chemotherapy of $\mathrm{S}-1$ for gastric cancer. Anticancer Res 38: 3745-3751, 2018.

33. Jin F, Han A, Shi F, Kong L and Yu J: The postoperative neutrophil-to-lymphocyte ratio and changes in this ratio predict survival after the complete resection of stage I non-small cell lung cancer. Onco Targets Ther 9: 6529-6537, 2016.

34. Albisinni S, Moussa I, Aoun F, Quackels T, Assenmacher G, Peltier $\mathrm{A}$ and Roumeguère $\mathrm{T}$ : The impact of postoperative inflammatory biomarkers on oncologic outcomes of bladder cancer. Prog Urol 29: 270-281, 2019.

35. Obermair A, Simunovic M, Isenring L and Janda M: Nutrition interventions in patients with gynecological cancers requiring surgery. Gynecol Oncol 145: 192-199, 2017.

36. Ballmer PE, Ochsenbein AF and Schütz-Hofmann S: Transcapillary escape rate of albumin positively correlates with plasma albumin concentration in acute but not in chronic inflammatory disease. Metabolism 43: 697-705, 1994.

37. Xiao Y, Ren YK, Cheng HJ, Wang L and Luo SX: Modified Glasgow prognostic score is an independent prognostic factor in patients with cervical cancer undergoing chemoradiotherapy. Int J Clin Exp Pathol 8: 5273-5281, 2015.

38. Ayhan A, Günakan E, Alyazıcı İ, Haberal N, Altunda ̆ Ö and Dursun P: The preoperative albumin level is an independent prognostic factor for optimally debulked epithelial ovarian cancer. Arch Gynecol Obstet 296: 989-995, 2017. International (CC BY-NC-ND 4.0) License. 